TRANSACTIONS OF THE

AMERICAN MATHEMATICAL SOCIETY

Volume 184, October 1973

\title{
CROSS-SECTIONS OF SYMPLECTIC STIEFEL MANIFOLDS
}

BY

\section{FRANÇOIS SIGRIST AND ULRICH SUTER}

ABSTRACT. The cross-section problem for the symplectic Stiefel manifolds is solved, using the now-proved Adams conjecture.

Introduction. Let $\mathrm{Sp}(n)$ be the symplectic group and $w_{n, k}=\mathrm{Sp}_{\mathrm{p}}(n) / \mathrm{Sp}(n-k)$ the symplectic Stiefel manifold. For $k>m$ one has an obvious map $p: W_{n, k} \rightarrow$ $W_{n, m}$, which is a fiber map with fiber $W_{n-m, k-m}$. The purpose of this paper is to give a complete description of the values of $n, k$ and $m$ for which the map $p$ has a cross-section.

The corresponding problem for the orthogonal Stiefel manifolds is already completely solved (Adams 1962, Eckmann-Whitehead 1963), as is the unitary case (Adams-Walker 1965, Suter 1966), the most famous contribution being Adams' solution of the vector field problem on spheres [1]. For the symplectic Stiefel manifolds, the explicit results previously known can be stated as follows:

(i) The map $p: w_{n, 2} \rightarrow W_{n, 1}=s^{4 n-1}$ has a cross-section if and only if $n$ is a multiple of 24 . This result is due to $I$. M. James [10].

(ii) For $k>m \geq 2$, the map $p: W_{n, k} \rightarrow W_{n, m}$ does not have a cross-section. For this result see [12, p. 203].

We shall dispose of the remaining cases, i.e. the cases with $m=1, k>2$, by the following theorem.

Theorem. The symplectic Stiefel fibring $p: W_{n, k} \rightarrow W_{n, 1}$ bas a cross-section if and only if one of the following two equivalent conditions bolds:

(I) For eacb integer $j$ with $0 \leq j \leq k-1$ the coefficient $a_{j}$ of $z^{j}$ in

$$
\left[\frac{2}{\sqrt{z}} \cdot \operatorname{sh}^{-1}\left(\frac{\sqrt{z}}{2}\right)\right]^{2 n}=\sum_{j=0}^{\infty} a_{j} z^{j}=1-\frac{n}{12} z+\cdots
$$

is an integer if $j$ is even and an even integer if $j$ is odd. $\left(\mathrm{sh}^{-1}\right.$ denotes the inverse of the byperbolic sine.)

(II) $n$ is a multiple of the integer $c_{k}$, called quaternionic James number, wbicb is defined by its decomposition into prime powers as follows:

Received by the editors November 16, 1972 and, in revised form, January 25, 1973 and February 20, 1973.

AMS (MOS) subject classifications (1970). Primary 55F50, 55E50.

Key words and phrases. Stiefel manifold, quaternionic projective space, James number, $K$-theory, $J$-homomorphism, Adams conjecture.

Copyright $\odot$ 1974, American Mathematical Society 


$$
\begin{array}{ll}
\nu_{2}\left(c_{k}\right)=\max _{s}\left(2 k-1,2 s+\nu_{2}(s)\right), & 1 \leq s \leq k-1, \\
\nu_{p}\left(c_{k}\right)=\max _{t}\left(t+\nu_{p}(t)\right), & 1 \leq t \leq\left[\frac{2 k-1}{p-1}\right], p \text { odd }<2 k, \\
\nu_{p}\left(c_{k}\right)=0, & p \text { odd }>2 k_{0} .
\end{array}
$$

(The integer $\nu_{p}(q)$ is the exponent of the prime $p$ in the prime power decomposition of q.)

We observe in particular that $c_{k}$ is divisible by all primes less than $2 k$. The number $c_{2}$ is equal to 24 , this is James' result mentioned above. Comparing $c_{k}$ with the (known) complex James number $b_{2 k}$ one obtains that $c_{k}$ is either equal to $b_{2 k}$ or to $1 / 2 b_{2 k^{\circ}}$ A closer study of the relation between $c_{k}$ and $b_{2 k}$ shows: for $k$ odd, $c_{k}$ is always equal to $1 / 2 b_{2 k}$. On the other hand if $k$ is even, there are cases with $c_{k}=b_{2 k}$ and such with $c_{k}=1 / 2 b_{2 k}$. In approximately $63 \%$ of all cases we have $c_{k}=1 / 2 b_{2 k}$.

The proof of the theorem is based on techniques developed by Adams and Walker in the unitary case [3]. Denoting by $K O(X)$ the real $K$-theory of a finite $\mathrm{CW}$-complex $X$ and by $J: K O(X) \rightarrow J(X)$ Atiyah's J-homomorphism, we state the following two theorems, which are the starting point of our study.

Theorem (James $[9,1.4])$. There exists a positive integer $c_{k}$, such that the Stiefel fibring $p: w_{n, k} \rightarrow S^{4 n-1}$ bas a cross-section if and only if $n$ is a multiple of $c_{k}$.

Theorem (Atiyah $[4,6.5])$. Let $\xi \in K O\left(H P^{k-1}\right)$ be the canonical real (4dimensional) Hopf bundle over the $(k-1)$-dimensional quaternionic projective , space $H P^{k-1}$. The James number $c_{k}$ is the order of $J(\xi)$ in the group $J\left(H P^{k-1}\right)$.

We first show that condition $I$ of our theorem expresses the fact that $n$ is a multiple of the order of $J(\xi)$ in $J\left(H P^{k-1}\right)$. This is done in $\S 1$ and $\$ 2$, using the group $J^{\prime}\left(H P^{k-1}\right)[3]$. (Using Quillens proof of the Adams conjecture [12] we get an isomorphism $J\left(H P^{k-1}\right) \cong J^{\prime}\left(H P^{k-1}\right)$.) In $\$ 3$ we then show that condition $I$ is equivalent to condition II. The final section is devoted to an investigation of the relationship between $c_{k}$ and $b_{2 k^{*}}$

1. Preliminaries. In this section we put together all the facts we need about ordinary cohomology and $K$-theory of the $q$-dimensional quaternionic right projective space $H P^{q}$.

Let $C P^{m}$ be the $m$-dimensional complex projective space. The classical Hopf map $S^{4 q+3} \rightarrow H P^{q}$ factors through $C P^{2 q+1}$ and gives rise to an $S^{2}$-fiber bundle 


$$
S^{2} \stackrel{i}{\rightarrow} C P^{2 q+1} \stackrel{g}{\rightarrow} H P^{q} .
$$

It will turn out that $g$ induces injections in ordinary cohomology as well as in $K$ theory. So we might regard $H^{*}\left(H P^{q} ; Z\right), H^{*}\left(H P^{q} ; Q\right), K U\left(H P^{q}\right)$ and $K O\left(H P^{q}\right)$ as subrings of the corresponding rings of $C P^{2 q+1}$, which are well known [3].

Let $b \in H^{2}\left(C P^{2 q+1} ; Z\right)$ be the canonical generator and let $a \in H^{4}\left(H P^{q} ; Z\right)$ be a generator; then for $R=Z$ or $Q$

$$
H^{*}\left(C P^{2 q+1} ; R\right)=R[b] \quad\left(\bmod b^{2 q+2}\right), \quad H^{*}\left(H P^{q} ; R\right)=R[a] \quad\left(\bmod a^{q+1}\right) .
$$

The fiber $S^{2}$ of $(1.0)$ is totally nonhomologous to zero (i.e. $i^{*}$ is onto). By the usual spectral-sequence argument, it follows that $g^{*}: H^{*}\left(H P^{q} ; R\right) \rightarrow$ $H^{*}\left(C P^{2 q+1} ; R\right)$ is injective and that we can choose the generator $a$ such that $g^{*}(a)=b^{2}$.

We write $\beta$ for the canonical complex line bundle over $C P^{2 q+1}$ and $\bar{\beta}$ for its complex-conjugate. The canonical quaternionic left line bundle over $H P^{q}$ is denoted by $\zeta$. (The bundle $\zeta$ is the dual of the quaternionic right line bundle associated to the principal $H^{*}$-bundle $\left(H^{q+1}-0\right) \rightarrow H P^{q}$; see $[4, \S 4]$.) Let $a$ be the 2-dimensional complex vector bundle underlying $\zeta$. For the induced bundle $\boldsymbol{g}^{*}(\alpha)$ one has

$$
g^{*}(\alpha) \cong \beta \oplus \bar{\beta}
$$

This fact is well known and can be proved by direct computation of the total spaces of the bundles involved (see also $[7,9.6]$ ).

We turn now to the computation of the complex $K$-theory of $H P^{q}$ and set

$$
\mu=\beta-1 \epsilon \tilde{K} U\left(C P^{2 q+1}\right), \quad \bar{\mu}=\bar{\beta}-1 \epsilon \widetilde{K} U\left(C P^{2 q+1}\right), \quad \nu=a-2 \epsilon \widetilde{K} U\left(H P^{q}\right) .
$$

By $[1,7.2]$ we have

$$
K U\left(C P^{2 q+1}\right)=Z[\mu]\left(\bmod \mu^{2 q+2}\right) .
$$

(1.4) Proposition. (i) The ring $K U\left(H P^{q}\right)$ is generated by $\nu$ subject to the relation $\nu^{q+1}=0$, i.e. $K U\left(H P^{q}\right)=Z[\nu]\left(\bmod \nu^{q+1}\right)$.

(ii) The homomorphism $g^{!}: K U\left(H P^{q}\right) \rightarrow K U\left(C P^{2 q+1}\right)$ is injective and given by $g^{!}(\nu)=\mu+\bar{\mu}$.

Proof. Since $H P^{q}$ is torsion free we get with [5] that $K U\left(H P^{q}\right)$ is a free abelian group of the same rank as $H^{\text {even }}\left(H P^{q} ; Z\right)$, which by $(1.1)$ is $q+1$.

With (1.2) we deduce $g^{!}(\nu)=\mu+\bar{\mu}=\mu^{2}+$ higher powers of $\mu\left(\bar{\mu}=-\mu+\mu^{2}-\right.$ 
$\ldots$, see $[1,7.2])$. Looking at $(1.3)$ we see that the elements $1, g^{!}(\nu), \cdots, g^{\prime}\left(\nu^{q}\right)$ generate a direct summand of rank $q+1$ in the free abelian group $K U\left(C P^{2 q+1}\right)$, and the proposition is proved.

Next we determine the real $K$-theory of $H P^{q}$. We shall prove that the complexification $c: K O\left(H P^{q}\right) \rightarrow K U\left(H P^{q}\right)$ is injective, i.e. $K O\left(H P^{q}\right)$ can be regarded as subring of $K U\left(H P^{q}\right)$.

To begin with, note that for the element $\nu=a-2 \epsilon \widetilde{K} U\left(H P^{q}\right)$ one has

$$
2 \nu, \nu^{2} \in c\left(\tilde{K} O\left(H P^{q}\right)\right)
$$

This is seen as follows. The bundle $a$ is by definition equal to $c^{\prime}(\zeta)$, where we write $c^{\prime}$ for the map associating to a quaternionic vector bundle its underlying complex vector bundle. Hence $a$ is self-conjugate, i.e. $a=\bar{a}$. Denoting by $r$ the realification of complex vector bundles we get $c(r(\alpha))=a \oplus \bar{\alpha}=2 a$ and deduce $2 \nu=c(r(\nu))$. The tensor product $\zeta \otimes \zeta$ is a real (4-dimensional) vector bundle and we have $c(\zeta \otimes \zeta)=c^{\prime}(\zeta) \otimes c^{\prime}(\zeta)=a \otimes a$. This implies $\nu^{2} \epsilon c\left(\widetilde{R} O\left(H P^{q}\right)\right)$. (A reference for the above remarks is $[8, \S 3]$.)

The following proposition contains the information we will need about the ring $K O\left(H P^{q}\right)$.

(1.6) Proposition. (i) The complexification bomomorphism c: $K O\left(H P^{q}\right) \rightarrow$ $K U\left(H P^{q}\right)$ is injective.

(ii) The subring $c\left(K O\left(H P^{q}\right)\right)$ of $K U\left(H P^{q}\right)$ is generated as an abelian group by the elements $1,2 \nu, \nu^{2}, \cdots, e_{j} \nu^{j}, \cdots, e_{q} \nu^{q}$, where the integer $e_{j}$ is equal to 1 if $j$ is even and equal to 2 if $j$ is odd.

Proof. Working with the cofibration $H P^{q-1} \stackrel{i}{\rightarrow} H P^{q} \stackrel{p}{\rightarrow} S^{4 q}$ we proceed by induction on $q$. For $H P^{1}=S^{4}$ the proposition is a consequence of $[6,(3.15)]$. We then consider the exact cohomology sequence

$$
\begin{array}{lcc}
\tilde{K} O^{-1}\left(S^{4 q}\right) \rightarrow \tilde{K} O^{-1}\left(H P^{q}\right) \rightarrow \tilde{K} O^{-1}\left(H P^{q-1}\right) & \delta \\
\quad R & \tilde{K} O\left(s^{4 q}\right) \rightarrow \tilde{K} O\left(H P^{q}\right) \rightarrow \tilde{K} O\left(H P^{q-1}\right) \stackrel{\delta}{\rightarrow} \tilde{K} O^{1}\left(s^{4 q}\right) \\
0 \text { or } Z_{2} & \mathbb{R} & 0
\end{array}
$$

and deduce first inductively, that $\tilde{K} O^{-1}\left(H P^{q}\right)$ is finite. This gives the short sequence $0 \rightarrow \widetilde{K} O\left(S^{4 q}\right) \rightarrow \widetilde{K} O\left(H P^{q}\right) \rightarrow \widetilde{K} O\left(H P^{q-1}\right) \rightarrow 0$ and by induction on $q$ one proves $\tilde{K} O\left(H P^{q}\right)$ is torsion free. The relation $r \circ^{\circ} c=2$ [8, Proposition 3.1] implies that $c$ is in jective. We turn now to the proof of (ii). The commutative diagram 


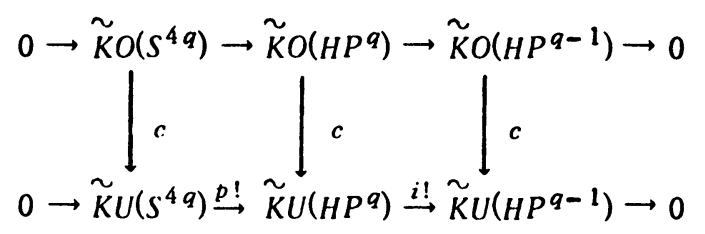

has exact rows and all the groups are free abelian. We write $\nu_{q}$ and $\nu_{q-1}$ for the generator of $\widetilde{K} U\left(H P^{q}\right)$ and $\widetilde{K} U\left(H P^{q-1}\right)$ respectively (see 1.4$)$. One has $i^{!}\left(\nu_{q}\right)=$ $\nu_{q-1}$. Let $e_{j}$ be equal to 1 if $j$ is even and equal to 2 if $j$ is odd. Then by (1.5) the elements $e_{j} \nu_{q}^{j}, j=1, \cdots, q$, belong to $c\left(\tilde{K} O\left(H P^{q}\right)\right)$. We have to show that these elements form a basis of $c\left(\tilde{K} O\left(H P^{q}\right)\right)$. The images $i^{!}\left(e_{j} \nu_{q}^{j}\right)=e_{j} \nu_{q-1}^{j}$, $j=1, \cdots, q-1$, are a basis of $c\left(\tilde{K} O\left(H P^{q-1}\right)\right)$ by inductive hypothesis and it remains to prove that $e_{q} \nu_{q}^{q}=p^{!}(c(\gamma))$ for a generator $\gamma \in \widetilde{K} O\left(S^{4 q}\right)=Z$. But $c(\gamma)=e_{q} \gamma_{C}$ for a suitable generator $\gamma_{C} \in \widetilde{K} U\left(S^{4 q}\right)$ (see $[8,(3.15)]$ ) and since ker $i^{!}=\operatorname{im} p^{!}$is generated by $\nu_{q}^{q}$ the proof is complete.

2. The J-calculation. The general reference for this section is the paper of Adams and Walker, On complex Stiefel manifolds [3].

Let $J\left(H P^{k-1}\right)$ be the group of equivalence classes of orthogonal sphere bundles over $H P^{k-1}$ with respect to stable fibre homotopy type and let $J: K O\left(H P^{k-1}\right) \rightarrow J\left(H P^{k-1}\right)$ be the canonical epimorphism [4]. According to Atiyah $[4,6.5]$ the fibration $W_{n, k} \rightarrow S^{4 n-1}$ has a cross-section if and only if in $J\left(H P^{k-1}\right)$ one has

$$
n \cdot J(r \alpha)=0
$$

where $r \alpha=r \circ c^{\prime}(\zeta)$ is the real vector bundle underlying the canonical quaternionic line bundle $\zeta$ (see $\$ 1$ ). To determine the integers $n$ which satisfy $(2.0)$ we use the groups $J^{\prime \prime}\left(H P^{k-1}\right)$ and $J^{\prime}\left(H P^{k-1}\right)$ defined in [3], which constitute a "computable" upper, respectively lower, bound of $J\left(H P^{k-1}\right)$. (Note in particular that we deal with the $J^{\prime}$ as defined in [3].)

(2.1) Lemma. For the space $H P^{q}$ one has the isomorphisms

$$
J^{\prime \prime}\left(H P^{q}\right) \stackrel{\theta^{\prime \prime}}{\cong} J\left(H P^{q}\right) \stackrel{\theta^{\prime}}{\cong} J^{\prime}\left(H P^{q}\right)
$$

Proof. The first isomorphism follows from the now-proved Adams conjecture [11]. To show that $\theta^{\prime} \circ \theta^{\prime \prime}$ is an isomorphism we copy the proof of [3, Lemma 6.1]. For $H P^{1}=S^{4}$, in general for $S^{4 q}$, we have $J^{\prime \prime}\left(S^{4 q}\right) \theta^{\prime} \cong \theta^{\prime \prime} J^{\prime}\left(S^{4 q}\right)$. (This fact follows in exactly the same way as the corresponding statement for the space $C P^{2 q} / C P^{2 q-2}$ (see $\left[3,4.10\right.$ and 5.3]).) The cofibration $H P^{q-1} \stackrel{i}{\rightarrow} H P^{q} \stackrel{p}{\rightarrow} S^{4 q}$ induces a commutative diagram: 


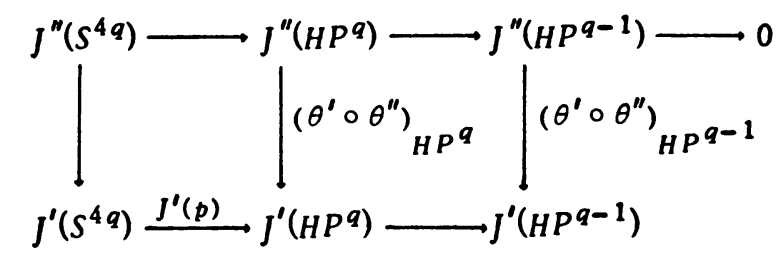

The upper row of this diagram is exact $[2,3.12], J^{\prime}(p)$ is a monomorphism $[3$, 4.9] and the verticals are epimorphisms. By induction on $q$ and easy diagramchasing one shows now that $\left(\theta^{\prime} \circ \theta^{n}\right)_{H P^{q}}$ is an isomorphism for all $q$ and the lemma is proved.

Lemma (2.1) enables us to work with $J^{\prime}$ instead of $J$. The condition (2.0) is satisfied if and only if $n \cdot J^{\prime}(r \alpha)=0$. By definition $[3, \S 4]$ we have $J^{\prime}\left(H P^{k-1}\right)=K O\left(H P^{k-1}\right) / V\left(H P^{k-1}\right)$, where $V\left(H P^{k-1}\right)$ is the subgroup of elements $\delta \in K O\left(H P^{k-1}\right)$, such that $\operatorname{sh}(\delta)=\mathrm{ch} \circ c(1+\gamma)$ for some element $\gamma \in \widetilde{K} O\left(H P^{k-1}\right)$. (Here sh: $K O(X) \rightarrow 1+\Sigma_{s>0}^{\infty} H^{4 s}(X ; Q)$ is the characteristic class corresponding to the power series of $\left(e^{y / 2}-e^{y / 2}\right) / y=(2 / y) \cdot \operatorname{sh}(y / 2)$, $c: K O(X) \rightarrow K U(X)$ is complexification and ch: $K U(X) \rightarrow H^{*}(X ; Q)$ is the Chern character.) The condition $n \cdot J^{\prime}(r \alpha)=J^{\prime}(n \cdot r \alpha)=0$ becomes then

$$
\operatorname{sh}(-n \cdot r a) \epsilon \operatorname{ch} \circ C\left(K O\left(H P^{k-1}\right)\right) \text {. }
$$

(We change $n$ to $-n$ for convenience.) Since $g^{*}: H^{*}\left(H P^{k-1} ; Q\right) \rightarrow H^{*}\left(C P^{2 k-1} ; Q\right)$ is injective (see $\$ 1$ ) we conclude

$$
n \cdot J(r \alpha)=0 \Leftrightarrow g^{*} \circ \operatorname{sh}(-n \cdot r \alpha) \in g^{*} \circ \operatorname{ch} \circ c\left(K O\left(H P^{k-1}\right)\right) .
$$

With (1.2) we compute $g^{*}(r \alpha)=r \circ g^{*}(\alpha)=r(\beta+\bar{\beta})=2 \cdot r \beta$. The naturality and the exponential property of sh imply $g^{*} \circ \operatorname{sh}(-n \cdot r \alpha)=\operatorname{sh}(-2 n \cdot r \beta)=$ $[\operatorname{sh}(r \beta)]^{-2 n}$. But $\operatorname{sh}(r \beta)=\left(e^{b / 2}-e^{-b / 2}\right) / b=(2 \cdot \operatorname{sh}(b / 2)) / b[3, \S 4]$ and hence

$$
g^{*} \circ \operatorname{sh}(-n \cdot r a)=\left[\frac{b}{2 \operatorname{sh}(b / 2)}\right]^{2 n} \in H^{*}\left(C P^{2 k-1} ; Q\right)=Q[b] \quad\left(\bmod b^{2 k}\right)
$$

(see 1.1). The image of the homomorphism $g^{*} \circ \mathrm{ch} \circ c=c h \circ g^{!} \circ c: K O\left(H P^{k-1}\right) \rightarrow$ $H^{*}\left(C P^{2 k-1} ; Q\right)$ is generated by

$$
e_{j} \cdot[2 \cdot \operatorname{sh}(b / 2)]^{2 j}\left(\bmod b^{2 k}\right)
$$

$$
j=0,1, \ldots, k-1, \text { where } e_{j}=\left\{\begin{array}{l}
1, \text { if } j \text { even, } \\
2, \text { if } j \text { odd. }
\end{array}\right.
$$

This follows from (1.6), (1.4) and $\operatorname{ch}(\mu+\bar{\mu})=\left(e^{b}-1+e^{-b}-1\right)=[2 \cdot \operatorname{sh}(b / 2)]^{2}$. 
In $Q[b]\left(\bmod b^{2 k}\right)$ one has a unique relation

$$
\left(R_{n}\right):\left[\frac{b}{2 \cdot \operatorname{sh}(b / 2)}\right]^{2 n}=\sum_{j=0}^{k-1} q_{j}(n) \cdot e_{j} \cdot\left[2 \cdot \operatorname{sh} \frac{b}{2}\right]^{2 j}\left(\bmod b^{2 k}\right)
$$

here $q_{0}(n)=1, q_{1}(n), \cdots, q_{k-1}(n)$ are rational numbers which depend on $n$. Consequently we deduce from (2.2), (2.3) and (2.4),

(2.5) Lemma. The following two conditions are equivalent.

(1) $n \cdot J(r a)=0$ in $J\left(H P^{k-1}\right)$.

(2) The coefficients $q_{0}(n)=1, q_{1}(n), \cdots, q_{k-1}(n)$ in $\left(R_{n}\right)$ are integers.

Substituting $b=2 \cdot \operatorname{sh}^{-1}(\sqrt{z} / 2)$ in $\left(R_{n}\right)$ we finally infer from (2.0) and (2.5) the following theorem, which is the first part of our main result.

(2.6) Theorem. The quaternionic Stiefel fibring $p: W_{n, k} \rightarrow S^{4 n-1}$ bas a cross-section if and only if for each integer $j$ with $0 \leq j \leq k-1$ the coefficient $a_{j}(n)$ of $z^{j}$ in

$$
\left(S_{n}\right):\left[\frac{2}{\sqrt{z}} \cdot \mathrm{sh}^{-1}\left(\frac{\sqrt{z}}{2}\right)\right]^{2 n}=\sum_{j=0}^{\infty} a_{j}(n) \cdot z^{j}
$$

is an integer if $j$ is even and an even integer if $j$ is odd.

3. Determination of the quaternionic James number. First let us fix some notation. For any rational number $q \neq 0$ and any prime $p$ we define the integer $\nu_{p}(q)$ by

$$
q=2^{\nu 2(q)} \cdot 3^{\nu} 3(q) \cdot 5^{\nu} s(q) \ldots
$$

i.e. $\nu_{p}(q)$ is the exponent of $p$ in the prime power decomposition of $q$. We set $\nu_{p}(0)=+\infty$ for all primes $p$.

According to our theorem (2.6) and the theorem of James [9] mentioned in the introduction, the quaternionic James number $c_{k}$ is the smallest integer $n$ such that for $j=0, \cdots, k-1$ one has

$$
\begin{aligned}
& \nu_{p}\left(a_{j}(n)\right) \geq 0, \quad \text { for } p \text { odd } \\
& \nu_{2}\left(a_{j}(n)\right) \geq \begin{cases}0, & \text { if } j \text { even, } \\
1, & \text { if } j \text { odd. }\end{cases}
\end{aligned}
$$

The following lemma is an essential step towards the computation of the odd part of $c_{k}$. 
(3.1) Lemma. Let $p$ be an odd prime. Then the following two conditions are equivalent.

(i) The first $k$ coefficients $a_{0}(n), \cdots, a_{k-1}(n)$ of $\left(S_{n}\right)$ satisfy $\nu_{p}\left(a_{i}(n)\right) \geq 0$.

(ii) The first $2 k$ coefficients $b_{0}(n), \cdots, b_{2 k-1}(n)$ of $\left[x^{-1} \log (1+x)\right]^{2 n}=$ $\Sigma_{i=0}^{\infty} b_{i}(n) \cdot x^{i}$ satisfy $\nu_{p}\left(b_{i}(n)\right) \geq 0$.

Proof. Setting $\sqrt{z} / 2=y$ we see that $\nu_{p}\left(a_{j}(n)\right) \geq 0, j=0, \cdots, k-1$, if and only if the coefficients $d_{i}(n), i=0, \cdots, 2 k-1$, of

$$
\sum_{i=1}^{\infty} d_{i}(n) \cdot y^{i}=\left[\frac{\operatorname{sh}^{-1}(y)}{y}\right]^{2 n}=\left[\frac{\log \left(y+\sqrt{1+y^{2}}\right)}{y}\right]^{2 n}
$$

satisfy $\nu_{p}\left(d_{i}(n)\right) \geq 0$.

The power series of $y+\sqrt{1+y^{2}}$ is of the form $1+g(y)$, where $g(y)$ has the inverse $b(x)=x-1 / 2 \Sigma_{i=2}^{\infty}(-1)^{i} x^{i}$. The coefficients of $b(x)$ and hence those of $g(y)$ are rational numbers having only powers of 2 in the denominator. Substituting $y=b(x)$ in $\Sigma_{i=0}^{\infty} d_{i}(n) \cdot y^{i+2 n}=[\log (1+g(y))]^{2 n}$ we deduce therefore by elementary manipulations of power series: one has $\nu_{p}\left(d_{i}(n)\right) \geq 0, i=0, \cdots, 2 k-1$, if and only if the coefficients $b_{i}(n), i=0, \cdots, 2 k-1$, of $\Sigma_{i=0}^{\infty} b_{i}(n) \cdot x^{i+2 n}=$ $[\log (1+x)]^{2 n}$ satisfy $\nu_{p}\left(b_{i}(n)\right) \geq 0$. This completes the proof of the lemma.

The power series of $\left[x^{-1} \log (1+x)\right]^{2 n}$ has been investigated by Atiyah and Todd [6] and the following proposition is a direct consequence of [6, Proposition 6.4] and our Lemma (3.1).

(3.2) Proposition. Let $p$ be an odd prime. Then the following two conditions are equivalent.

(i) The first $k$ coefficients $a_{0}(n), \cdots, a_{k-1}(n)$ of $\left(S_{n}\right)$ satisfy $\nu_{p}\left(a_{j}(n)\right) \geq 0$.

$$
\nu_{p}(n) \geq \begin{cases}\max \left(r+\nu_{p}(r)\right), & 1 \leq r \leq[(2 k-1) /(p-1)], p \leq 2 k-1, \\ 0, & p>2 k .\end{cases}
$$

Next we deal with the prime 2 and show

(3.3) Proposition. The following two conditions are equivalent.

(i) The first $k$ coefficients $a_{0}(n), \cdots, a_{k-1}(n)$ of $\left(S_{n}\right)$ satisfy

$$
\nu_{2}\left(a_{j}(n)\right) \geq\left\{\begin{array}{l}
0, \text { if } j \text { even, } \\
1, \text { if } j \text { odd. }
\end{array}\right.
$$


(ii) $\nu_{2}(n) \geq \max _{s}\left(2 k-1,2 s+\nu_{2}(s)\right), 1 \leq s \leq k-1$.

We postpone the proof of (3.3) for a moment. Referring to (3.0), (3.2) and (3.3) we obtain the following theorem, which constitutes the second part of our main result stated in the introduction.

(3.4) Theorem. The quaternionic James number $c_{k}$ is determined by

$$
\begin{aligned}
& \nu_{2}\left(c_{k}\right)=\max _{s}\left(2 k-1,2 s+\nu_{2}(s)\right), \quad 1 \leq s \leq k-1, \\
& \nu_{p}\left(c_{k}\right)=\max _{r}\left(r+\nu_{p}(r)\right), \quad 1 \leq r \leq\left[\frac{2 k-1}{p-1}\right], p \text { odd }<2 k, \\
& \nu_{p}\left(c_{k}\right)=0, \quad p \text { odd }>2 k .
\end{aligned}
$$

Now we turn to the proof of (3.3). To begin with we provide two lemmas.

(3.5) Lemma. Suppose that $\nu_{2}(n) \geq 2 j-1$. Then for the binomial coefficient $\left(\begin{array}{l}n \\ j\end{array}\right)$ one has

$$
\nu_{2}\left(\begin{array}{l}
n \\
j
\end{array}\right)=\nu_{2}(n)-\nu_{2}(j)
$$

Proof. Write

$$
\left(\begin{array}{l}
n \\
j
\end{array}\right)=\frac{n}{j} \cdot \frac{n-1}{1} \cdot \frac{n-2}{2} \cdot \ldots \cdot \frac{n-(j-1)}{j-1}
$$

and note that $\nu_{2}((n-q) / q)=0$ for $1 \leq q<2^{2 j-1}$.

Next we observe that the function $f(z)=\left[(2 / \sqrt{z}) \cdot \operatorname{sh}^{-1}(\sqrt{z} / 2)\right]^{2 n}$ satisfies the functional equation

$$
f(z)=(1+z / 4)^{n} \cdot f\left(4 z+z^{2}\right) .
$$

This implies the following relation for the coefficients $a_{0}(n)=1, a_{1}(n), \cdots$, $a_{j}(n), \cdots$ of $\left(S_{n}\right)$ (see 2.6).

$$
\left(\begin{array}{l}
n \\
j
\end{array}\right)+\sum_{i=1}^{i-1}\left(\begin{array}{c}
n+i \\
j-i
\end{array}\right) 4^{2 i} a_{i}(n)=4^{j}\left(1-4^{j}\right) a_{j}(n), \quad j=1,2, \ldots
$$

Working with the equations (3.6) we will prove

(3.7) Lemma. If $\nu_{2}(n) \geq 2 j-1$, then 


$$
\nu_{2}(n)=2 j+\nu_{2}(j)+\nu_{2}\left(a_{j}(n)\right)
$$

Proof. The proof is by induction over $j$. For $j=1$ we have $a_{1}(n)=-n / 12$ and hence $\nu_{2}\left(a_{1}(n)\right)=\nu_{2}(n)-2$.

Now let $j>1$ and assume $\nu_{2}(n) \geq 2 j-1$. This means in particular that $\nu_{2}(n) \geq 2 i-1, i=1, \cdots, j-1$, and one has by inductive hypothesis that $\nu_{2}\left(a_{i}(n)\right)=\nu_{2}(n)-2 i-\nu_{2}(i), i=1, \cdots, j=1$. One computes then

$$
\begin{aligned}
\nu_{2}\left(\begin{array}{c}
n+i \\
j-i
\end{array}\right) 4^{2 i} a_{i}(n) & \geq 4 i+\nu_{2}\left(a_{i}(n)\right)=\nu_{2}(n)+2 i-\nu_{2}(i) \\
& >\nu_{2}(n)+1, \quad i=1, \cdots, j-1 .
\end{aligned}
$$

From Lemma (3.5) we get $\nu_{2}\left(\begin{array}{l}n \\ j\end{array}\right)=\nu_{2}(n)-\nu_{2}(j)<\nu_{2}(n)+1$ and deduce with the equation

$$
\left(\begin{array}{c}
n \\
j
\end{array}\right)+\sum_{i=1}^{j-1}\left(\begin{array}{c}
n+i \\
j-i
\end{array}\right) 4^{2 i} a_{i}(n)=4^{j}\left(1-4^{j}\right) a_{j}(n)
$$

that $\nu_{2}(n)-\nu_{2}(j)=2 j+\nu_{2}\left(a_{j}(n)\right)$. Q.E.D.

Proof of Proposition (3.3). We first show that (ii) implies (i). If $\nu_{2}(n)=$ $\max _{1 \leq s \leq k-1}\left(2 k-1,2 s+\nu_{2}(s)\right)$ then one has in particular $\nu_{2}(n) \geq$ $\max \left(2 j+1,2 j+\nu_{2}(j)\right)>2 j-1, j=1, \cdots, k-1$, and with Lemma (3.7) one derives readily

$$
\nu_{2}\left(a_{j}(n)\right) \geq \begin{cases}0, & \text { if } j \text { even, } \\ 1, & \text { if } j \text { odd },\end{cases}
$$

To prove (i) $\Rightarrow$ (ii) we proceed by induction on $k$. If $k=2$, then $\nu_{2}\left(a_{1}(n)\right)=$ $\nu_{2}(-n / 12) \geq 1$ means precisely $\nu_{2}(n) \geq 3=\max (3,2)$. Now let $k>2$ and assume

$$
\nu_{2}\left(a_{j}(n)\right) \geq\left\{\begin{array}{l}
0, \text { if } j \text { even, } \\
1, \text { if } j \text { odd, }
\end{array} \quad j=0,1, \cdots, k-1, k .\right.
$$

By inductive hypothesis we have

$$
\nu_{2}(n) \geq \max _{1 \leq s \leq k-1}\left(2 k-1,2 s+\nu_{2}(s)\right) \geq 2 k-1 .
$$

With Lemma (3.7) we conclude

$$
\nu_{2}(n)=2 k+\nu_{2}(k)+\nu_{2}\left(a_{k}(n)\right) \geq \begin{cases}2 k+\nu_{2}(k), & \text { if } k \text { even, } \\ 2 k+1, & \text { if } k \text { odd. }\end{cases}
$$


Hence $\nu_{2}(n) \geq \max _{1 \leq s \leq k}\left(2 k+1,2 s+\nu_{2}(s)\right)$.

4. Comparison between the complex and the quaternionic case. In this section we study the relation between $c_{k}$ and the known complex James number $b_{2 k}[3$, Theorem 1.2].

(4.0) Proposition. The quaternionic James number $c_{k}$ is either equal to the complex James number $b_{2 k}$ or to $1 / 2 b_{2 k}$.

Proof. The proposition follows at once from Theorem (3.4), $[3,1.2]$ and $[6,1.7]$.

We give a second proof which does not depend on our calculations in $\$ 3$, using the functor $J^{\prime \prime}$. First we show that the element $J_{C}^{\prime \prime}(\alpha) \in J_{C}^{\prime \prime}\left(H P^{k-1}\right)$ has the same order as $J^{\prime \prime}(r \beta) \in J^{\prime \prime}\left(C P^{2 k-2}\right)$ (notation as in $\S_{1}$ and $\S 2$ ). The homomorphism $g_{1}^{*}: K U\left(H P^{k-1}\right) \rightarrow K U\left(C P^{2 k-2}\right)$ induced by $C P^{2 k-2} \rightarrow C P^{2 k-1} \stackrel{g}{\rightarrow} H P^{k-1}$ is given by $g_{1}^{*}(\alpha)=\beta+\bar{\beta}$ (see 1.2) and the complexification $c: K O\left(C P^{2 k-2}\right) \rightarrow$ $K U\left(C P^{2 k-2}\right)$ is determined by $c(r \beta)=\beta+\bar{\beta}$. Since both $g_{1}^{*}$ and $c$ are injective (see (1.5) and [3, 2.2(iv)]) and compatible with the $\psi$-operations one obtains, slightly abusing notation, a $\psi \cdot$-ring isomorphism $c^{-1} \circ g_{1}^{*}=B: K U\left(H P^{k-1}\right) \cong$ $K O\left(C P^{2 k-2}\right) .(1)$ With the induced isomorphism $J^{\prime \prime}(B): J_{C}^{\prime \prime}\left(H P^{k-1}\right) \cong J^{\prime \prime}\left(C P^{2 k-2}\right)$ (see [2]) we deduce : the order of $J_{C}^{\prime \prime}(\alpha)$ is equal to the order of $J^{\prime \prime}(r \beta)$ which is $b_{2 k-1}$ [3]; by [6, p. 344] one has $b_{2 k-1}=b_{2 k^{*}}$

The homomorphism $J^{\prime \prime}(r): J_{C}^{\prime \prime}\left(H P^{k-1}\right) \rightarrow J^{\prime \prime}\left(H P^{k-1}\right)$ (see [3, Appendix]) maps $J_{C}^{\prime \prime}(\alpha)$ onto $J^{\prime \prime}(r \alpha)$ and it follows, that the order of $J^{\prime \prime}(r \alpha)$, i.e. the integer $c_{k}$, divides $b_{2 k}$. But $b_{2 k}$ is a factor of $2 c_{k}[10,1.5]$ and $(4.0)$ is proved.

Next we give a description of the distribution of the integers $k$ for which $c_{k}=1 / 2 b_{2 k}$ and an approach to the evaluation of the corresponding density. We have $c_{1}=1 / 2 b_{2}$. If $k \geq 2$ we get the following result:

(4.1) Theorem. Let $k \geq 2$. Then one bas $c_{k}=1 / 2 b_{2 k}$ if and only if the integer $k$ belongs to the set

$$
A=\left\{s+n \cdot 2^{2 s-1} \mid s, n \in N\right\} .
$$

(By $N$ we denote the set of integers $\geq 1$. )

Before giving the proof, let us draw some simple consequences illustrating the result.

(a) Given an integer $k$, it can be decided in a finite number of steps whether $c_{k}=1 / 2 b_{2 k}$. This is obvious from the description of $A$.

(1) This elegant way of deriving the $\psi$-ring isomorphism $K U\left(H P^{k-1}\right) \simeq K O\left(C P^{2 k-2}\right)$ was communicated to us by J. F. Adams. 
(b) If $k$ is odd, $c_{k}=1 / 2 b_{2 k}$. Indeed, all odd integers belong to $A$.

(c) The first even value of $k$ for which $c_{k}=1 / 2 b_{2 k}$ is $k=10$.

Proof of Theorem (4.1). We first recall from Theorem (3.4) that

$$
\nu_{2}\left(c_{k}\right)=\max _{1 \leq t \leq k-1}\left(2 k-1,2 t+\nu_{2}(t)\right)=\max _{1 \leq r \leq 2 k-1}\left(2 k-1, r+\nu_{2}(r)-1\right) .
$$

By $[3,1.2]$ and $[6,1.7]$ one has

$$
\nu_{2}\left(b_{2 k}\right)=\max _{1 \leq r \leq 2 k-1}\left(r+\nu_{2}(r)\right)
$$

We then show that the following four properties of an integer $k \geq 2$ are equivalent.

(i) $c_{k}=1 / 2 b_{2 k}$.

(ii) $\nu_{2}\left(b_{2 k}\right)>2 k-1$.

(iii) There exists an integer $s$ with $0<s<k$ and $k \equiv s\left(\bmod 2^{2 s-1}\right)$.

(iv) $k \in A$.

The equivalence (i) $\Leftrightarrow$ (ii) follows immediately from the comparison of $\nu_{2}\left(c_{k}\right)$ and $\nu_{2}\left(b_{2 k}\right)$. The equivalence (iii) $\Leftrightarrow$ (iv) is trivial.

(ii) $\rightarrow$ (iii). If $\nu_{2}\left(b_{2 k}\right)>2 k-1$ there exists an even integer $r$ with $1 \leq r \leq$ $2 k-1$ and $r+\nu_{2}(r)>2 k-1$. Putting $r=2 k-2 s$ we obtain the existence of $s$ with $0<s<k$ and $2 k-2 s+\nu_{2}(2 k-2 s)>2 k-1$. This last condition is equivalent to $k-s \equiv 0\left(\bmod 2^{2 s-1}\right)$.

(iii) $\rightarrow$ (ii). Taking $r=2 k-2 s$ we get

$$
\nu_{2}\left(b_{2 k}\right) \geq(2 k-2 s)+\nu_{2}(2 k-2 s) \geq(2 k-2 s)+2 s>2 k-1 .
$$

This proves the theorem.

We now turn to an evaluation of the density of $A$ in $N$. We are greatly indebted to our friend H. Carnal for the considerations below. Any integer of $A$ belongs to exactly one arithmetic progression $A_{q}=\left\{q+r \cdot 2^{2 q-1}\right\}$ with $q \notin A$ (!). Moreover the set $A$ is the disjoint union $A=\bigcup_{q \notin A} A_{q}$ and its density $\alpha$ is equal to $\Sigma_{m \& A} 2^{-(2 m-1)}$. We then write

$$
\begin{aligned}
\alpha & =\sum_{m \in A} 2^{-(2 m-1)}=\sum_{m \in N} 2^{-(2 m-1)}-\sum_{m \in A} 2^{-(2 m-1)}=\frac{2}{3}-\sum_{n \notin A} \sum_{m \in A_{n}} 2^{-(2 m-1)} \\
& =\frac{2}{3}-\sum_{n \notin A} \sum_{m=1}^{\infty} 2^{-(2 n-1)-m \cdot 2^{2 n}}=\frac{2}{3}-\sum_{n \in A} 2^{-(2 n-1)\left(2^{\left(2^{2 n}\right)}-1\right)^{-1}} \\
& =\frac{2}{3}-\frac{1}{2\left(2^{4}-1\right)}+\frac{1}{2^{3}\left(2^{16}-1\right)}+\frac{1}{2^{7}\left(2^{256}-1\right)} \cdots
\end{aligned}
$$


The convergence of the series is extraordinarily rapid. Using the fact that $A$ contains sequences of consecutive integers of arbitrary length, one deduces easily from Liouville's criterion that $\alpha$ is a transcendental number.

The approximate value of $a$ is 0.63 . Hence in about $63 \%$ of all cases, the James number $c_{k}$ is equal to $1 / 2 b_{2 k}$ ("Complex vector fields on spheres can be chosen quaternionic".) It remains interesting to ask what geometric (and homotopy-theoretic) phenomena could be related to this strange algebraic fact.

\section{REFERENCES}

1. J. F. Adams, Vector fields on spheres, Ann. of Math. (2) 75 (1962), 603-632. MR 25 \#2614.

2. - On the groups $J(X)$. II, Topology 3 (1965), 137-171. MR $33 \# 6626$.

3. J. F. Adams and G. Walker, On complex Stiefel manifolds, Proc. Cambridge Philos. Soc. 61 (1965), 81-103. MR 30 \#1516.

4. M. F. Atiyah, Thom complexes, Proc. London Math. Soc. (3) 11 (1961), 291-310. MR 24 \#A1727.

5. M. F. Atiyah and F. Hirzebruch, Vector bundles and homogeneous spaces, Proc. Sympos. Pure Math., vol. 3, Amer. Math. Soc., Providence, R. I., pp. 7-38. MR 25 \#2617.

6. M. F. Atiyah and J. A. Todd, On complex Stiefel manifolds, Proc. Cambridge Philos. Soc. 56 (1960), 342-353. MR 24 \#A2392.

7. A. Borel and F. Hirzebruch, Characteristic classes and homogeneous spaces. I, Amer. J. Math. 80 (1958), 458-538. MR 21 \#1586.

8. R. Bott, Quelques remarques sur les théorèmes de périodicité, Bull. Soc. Math. France 87 (1959), 293-310. MR 23 \#A3577.

9. I. M. James, Cross-sections of Stiefel manifolds, Proc. London Math. Soc. (3) 8 (1958), 536-547. MR $20 \# 7268$.

10. - Spaces associated with Stiefel manifolds, Proc. London Math. Soc. (3) 9 (1959), 115-140. MR $21 \# 1596$.

11. D. Quillen, The Adams conjecture, Topology 10 (1970), 67-80. MR 43 \#5525.

12. U. Suter, Die Nicht-Existenz von Schnittflächen komplexer Stiefel-Mannigfaltigkeiten, Math. Z. 113 (1970), 196-204. MR $42 \# 1137$.

INSTITUT DE MATHÉMATIQUES, UNIVERSITÉ DE NEUCHÂTEL, NEUCHÂTEL, SWITZERLAND

DEPARTMENT OF MATHEMATICS, UNIVERSITY OF BRITISH COLUMBIA, VANCOUVER, BRITISH COLUMBIA, CANADA 